\title{
THREE NEW CONTACT BINARIES NEAR THE CENTRE OF NGC 6121 (M4)
}

\author{
J. W. MENZIES \\ South African Astronomical Observatory \\ $P O$ Box 9, Observatory 7935, S. Africa
}

The central $2 \times 3 \operatorname{arcmin}^{2}$ region of M4 has been observed as part of a programme to find blue stragglers in globular clusters. A preliminary analysis has been carried out of $58 \mathrm{~V}$ frames and $65 \mathrm{I}$ frames obtained on two separate occasions with an RCA CCD on the $1.0 \mathrm{~m}$ telescope at Sutherland.

Photometry was performed with DAOPHOT in the IRAF environment. The colour-magnitude diagram based on 2 frames in each of $B$ and $V$ shows a significant number of blue stragglers and a clump of possible 'yellow' stragglers.

The 7 known RR Lyraes on the frames were confirmed. Three new contact systems were found. Because the time coverage is only $5.3 \mathrm{~h}$ and $4.3 \mathrm{~h}$ on two nights separated by 56 days, periods cannot be determined from the light curves, but they are probably of the order of 0.3 to 0.4 day. One of the stars is clearly a blue straggler; the second may comprise a pair of main sequence stars since, in the colour-magnitude diagram, it lies amongst the stars that have just evolved beyond the turnoff region; the third variable is rather red, and lies below the subgiant branch, to the red of the main sequence.

The suspected variable reported by Yao (1993) appears to be variable in the $\mathrm{V}$ series, but more or less constant in the I series. It has a fainter red companion only about 2 arcsec away, which causes problems for photometry in poorer seeing. More observations are required to determine a period for the star; Yao found 2 periods, neither of which is confirmed by the present data.

\section{References}

Yao, B.-A. (1993) IBVS 3846. 